\title{
A new apparent quasar pair: Q2225-403A,B
}

\author{
R. Decarli, ${ }^{1 \star}$ A. Treves ${ }^{1}$ and R. Falomo ${ }^{2}$ \\ ${ }^{1}$ Università degli Studi dell'Insubria, via Valleggio 11, 22100 Como, Italy \\ ${ }^{2}$ INAF - Osservatorio Astronomico di Padova, Vicolo dell'Osservatorio 5, 35122 Padova, Italy
}

Accepted 2009 March 9. Received 2009 March 2; in original form 2009 February 2

\begin{abstract}
We report the serendipitous discovery of a previously unknown quasar at 10.5 arcsec from

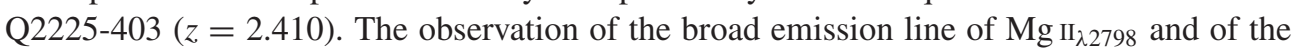
surrounding Fe II multiplets indicates that the companion quasar is at $z=0.932$. The spectrum of Q2225-403 shows a number of absorption lines, the most noteworthy is the $\mathrm{Mg}$ II line at the same redshift of the companion, suggesting that we are probing the gas within the halo $\sim 80 \mathrm{kpc}$ from the closer quasar. From high-resolution near-infrared images, we were able to resolve the host galaxies of the two quasars. Basing on the known surface density of quasars in the $2 \mathrm{dF}$ survey, we estimate that the probability of finding such a close pair is $\lesssim 1$ per cent.
\end{abstract}

Key words: galaxies: active - quasars: general - quasars: individual: Q2225-403.

\section{INTRODUCTION}

Quasar pairs can be classified in physical pairs, gravitational lenses and projected associations. Quasars in physical pairs are gravitationally interacting or belong to the same structure (e.g. a cluster of galaxies). They represent a formidable tool to improve our understanding of the evolution of galaxy and dark matter clustering with Cosmic Time, since they can be traced up to very high redshift (Komberg, Kravtsov \& Lukash 1996; Shen et al. 2008). They can also provide information about the role of galaxy interactions in triggering nuclear activity (e.g. Foreman, Volonteri \& Dotti 2009; Kang \& Im 2009). Gravitationally lensed quasars allow an unparalleled insight of the lens distribution of matter (e.g. Wittman et al. 2000; Chieregato, Miranda \& Jetzer 2007). Projected pairs can be used as probes of the spatial structure and ionization properties of intervening intergalactic medium (e.g. Jakobsen et al. 1986; D'Odorico et al. 2008; Gallerani et al. 2008) and, through the transverse proximity effect (Schirber, Miralda-Escudé \& McDonald 2004), of the megayear variability and duty cycle of quasars.

Up to now, only a dozen of apparent quasar pairs with angular separation less than 10 arcsec are known. Recent large field surveys, such as the Sloan Digital Sky Survey (SDSS; Adelman-McCarthy et al. 2008), collected spectra of $\sim 100000$ quasars and probed their large-scale $(>0.5 \mathrm{Mpc})$ clustering. Nevertheless, the limit due to the finite physical dimension of the spectroscopic fibres prevented the observation of objects with angular separations less than 55 arcsec, making this survey unsuitable for finding quasar pairs.

In the framework of the study of the $M_{\mathrm{BH}}-L_{\mathrm{host}}$ relation throughout Cosmic Time (Decarli et al., in preparation), we collected highresolution near-infrared (NIR) imaging (Kotilainen et al. 2009, hereafter K09) and optical spectroscopy (Decarli et al., in preparation)

\footnotetext{
${ }^{\star}$ E-mail: roberto.decarli@mib.infn.it
}

of Q2225-403 (hereafter, quasar A), a $z=2.410$ quasar first reported by Hewitt \& Burbidge (1993). We set the slit orientation so that we simultaneously observed both quasar A and the 10.5 arcsec north-east source with similar magnitude (see Fig. 1). The spectrum of the companion shows it is a quasar at $z=0.932$ (quasar B). In this Letter, we discuss the properties of this system together with a statistical analysis of apparent quasar pairs.

Throughout the Letter, we adopt a concordance cosmology with $H_{0}=70 \mathrm{~km} \mathrm{~s}^{-1} \mathrm{Mpc}^{-1}, \Omega_{m}=0.3, \Omega_{\Lambda}=0.7$.

\section{OBSERVATIONS AND DATA REDUCTION}

\subsection{Spectroscopic data}

Spectroscopy was collected at the European Southern Observatory (ESO) 3.6-m telescope in La Silla [Chile; program ID: 079.B0304(A)] on 2007 September 9 (Decarli et al., in preparation). Spectra were obtained through a 1.2 -arcsec slit in the wavelength 4100-7500 ̊ with a spectral resolution $R \sim 400$. Standard recipes for data reduction where adopted. Three individual exposures, for a total of 71-m integration time, were acquired. We set the position angle to 131.6, in order to observe simultaneously both Q2225-403 A and B. Two-dimensional spectra were bias subtracted, flat-fielded, re-aligned and combined scaling according to signal-to-noise ratio. One-dimensional spectra were extracted and wavelength- and flux-calibrated. The same calibration procedures were adopted for the two spectra. An absolute flux calibration was performed using corollary $R$-band photometry (see Fig. 1, left-hand panel). Final spectra were then de-reddened according to the $E(B-V)$ maps from Schlegel, Finkbeiner \& Davis (1998).

\subsection{Imaging data}

A deep Ks-band image of Q2225-403 field was obtained using the Infrared Spectrometer and Array Camera (ISAAC; Moorwood et al. 


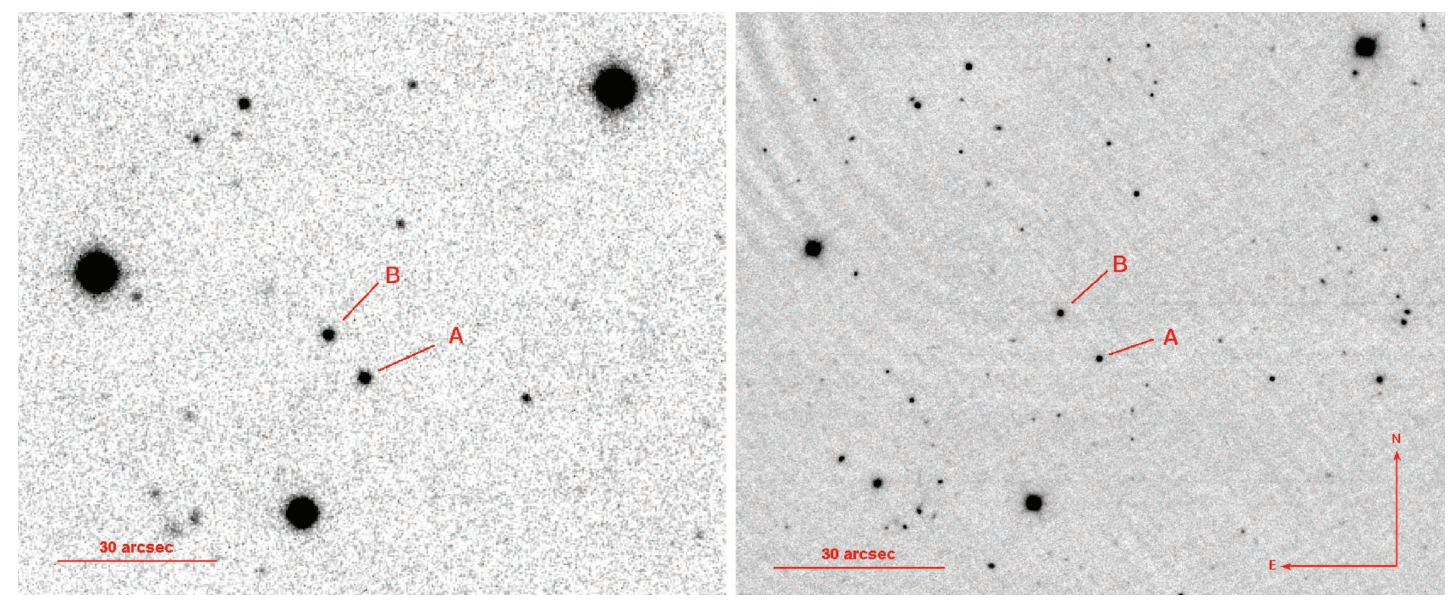

Figure 1. The field of quasar pair Q2225-403A,B in $R$ (left-hand panel), observed at the ESO/3.6-m telescope and in $K_{\mathrm{S}}$ (right-hand panel) taken at the ESO/VLT. 'A' marks the original quasar at $z=2.410$, 'B' refers to the new $z=0.932$ quasar.

1998), mounted on UT1 (Antu) of the ESO Very Large Telescope (VLT; see Fig. 1, right-hand panel). Q2225-403 was part of a sample of 16 objects, selected from the VCV06 catalogue in order to have relatively faint nuclear absolute magnitudes $\left(-26>M_{V}>\right.$ $-27), 2<z<3$ and $2-3$ bright stars in the close field in order to accurately characterize the point spread function (this is mandatory for the study of the host galaxies of bright quasars). We refer to K09 for details on the observations and data reduction. The average seeing was $0.46 \pm 0.05$ arcsec and the sky brightness was 13.34 mag $\operatorname{arcsec}^{-2}$. Photometric calibration was performed through the comparison with Two-Micron All-Sky Survey magnitudes of bright stars available in the field. The estimated photometric accuracy is $0.05 \mathrm{mag}$.

\section{RESULTS}

Quasar B ( $\left(\mathrm{RA}_{\mathrm{J} 2000}=22^{\mathrm{h}} 28^{\mathrm{m}} 50^{\mathrm{s}} .4\right.$; Dec. $\left.\mathrm{J} 2000=-40^{\circ} 08^{\prime} 27^{\prime \prime}\right)$ is not present in the catalogue by Veron-Cetty \& Veron (2006, hereafter VCV06), or in the NASA/IPAC Extragalactic Database (NED) or SIMBAD archives. It appears in the USNO-B1.0 catalogue (source ID: 0498-0814048, $m_{R}=20.0, m_{\mathrm{B}}=20.4$, grossly consistent with the photometry of our $R$-band image, $m=19.6$ ). The optical spectrum clearly shows the broad $\mathrm{Mg}$ II line surrounded by Fe II multiplets (see Fig. 2), leaving no doubt on the source nature. The peak of the $\mathrm{Mg}$ II line is observed at $5406 \AA$, yielding $z=0.932$.

A number of absorption systems are apparent in the spectra of Q2225-403A,B (see Table 1). In quasar B, an absorption at 7393 $\AA$ is detected, that is consistent with the $\mathrm{Fe}_{3830}$, typical of earlytype galaxies, at $z=0.932$. Other absorption lines are detected in the spectrum of quasar A at $4547 \AA$ and B at $4661 \AA$. Assuming

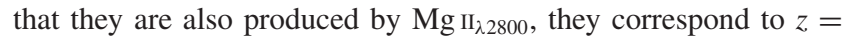
0.625 and 0.665 , respectively. Since each feature is observed only in one spectrum, we argue that the amount of intervening gas of the two clouds drops in a relatively small spatial scale $(\lesssim 75 \mathrm{kpc})$. Furthermore, in the spectrum of quasar A a faint feature is present at $5408 \AA$ (see Fig. 2). This absorption is clearly present in each of the three individual exposures of the spectrum. Therefore, we are confident that it is a real feature. The most likely identification of this line is with the $\operatorname{Mg}$ II doublet $(\lambda \lambda=2796,2804 \AA)$, at the same redshift of quasar $\mathrm{B}, z=0.932$. At the resolution of our observations, the two components of the doublet are blended. The presence of this absorption reveals an extended halo around the host galaxy of quasar B at the projected distance of $83 \mathrm{kpc} .{ }^{1}$

From the analysis of NIR imaging data, we are able to detect the host galaxy of both quasars. For quasar B, the $K_{\mathrm{s}}$ band roughly corresponds to the rest-frame $J$. We convert the observed magnitude into $R$ band by assuming the elliptical galaxy template by Mannucci et al. (2001), yielding $M_{R}$ (host) $=-23.2$. The galaxy is well resolved and modelled with a de Vaucouleurs profile with scale radius $R_{\text {eff }}=5.2 \mathrm{kpc}$ (see Fig. 3). The nuclear component has $M_{R}($ nuc $)=-22.5$. Assuming the quasar template in Francis et al. (1991), we infer $R-i=-0.04$. The derived $i$-band nuclear magnitude places source $B$ at the faint end of quasar luminosity function, but matching the usual $M_{i}<-22$ criterion for quasar classification (e.g. Richards et al. 2006).

In a study of quasar-galaxy projected associations, Kacprzak et al. (2007) found a correlation between the equivalent width (EW) of $\mathrm{Mg} \mathrm{II}_{\lambda 2796}$ and the asymmetry of the galaxy, suggesting a connection between the intervening metal absorption systems and the properties of the galaxy environment. According to this relationship, the stronger are the absorptions, the more disturbed is the morphology of the galaxy. In our case, we are unable to resolve the $\mathrm{Mg} \mathrm{I}_{\lambda 2796}$ line. If we assume a ratio between the two components of the $\mathrm{Mg}$ II doublet of 1.7 , we infer $\mathrm{Mg}_{\mathrm{I}_{\lambda 2796}}=0.8$. We note that a weak indication of asymmetry is apparent in the faintest surface brightness level of the host galaxy of quasar B, that is in qualitative agreement with the trend suggested by Kacprzak et al. (2007).

\section{AN INVENTORY OF APPARENT QUASAR PAIRS}

Due to the importance of quasar pairs of the type discussed here, we made an inventory of similar systems starting from the VCV06 catalogue. Out of $\sim 85000$ quasars, we found 19 pairs with angular separation $\theta<10$ arcsec and line-of-sight velocity differences exceeding $3000 \mathrm{~km} \mathrm{~s}^{-1}$ (hence excluding physical pairs). They are listed in Table 2. We note that only 11 systems out of 19 have been already considered in the framework of quasar pairs. On average, apparent pairs reported in Table 2 have $\langle z$ (near) $\rangle \approx 1.4$ and

\footnotetext{
${ }^{1}$ For similar cases see Bowen et al. (2007), Hennawi \& Prochaska (2007) and Prochaska \& Hennawi (2009).
} 


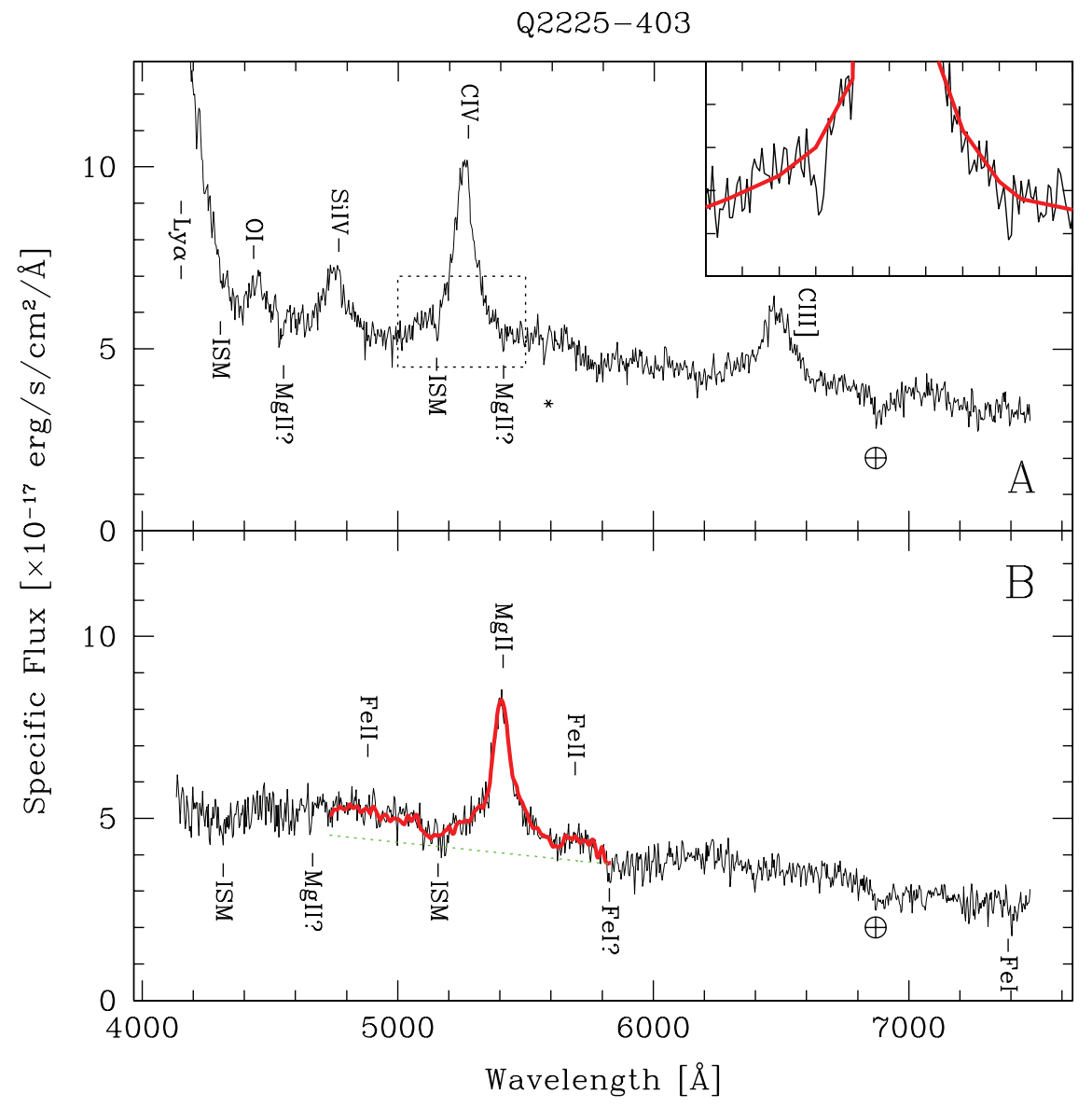

Figure 2. Spectra of quasars Q2225-403 A and B. Main emission and absorption lines are labelled (see also Table 1). The $\oplus$ symbols mark main atmospheric absorptions. The asterisk refers to the residual of the strong night sky line at $5577 \AA \AA$. ISM labels the features due to the Galactic interstellar medium. Top panel - the inset in the upper panel highlights the 5000-5500 $\AA$ region, with the absorption feature of $\mathrm{Mg}$ II at the bottom of the broad C IV emission line. The bold line represent the fit of the $\mathrm{C}_{\text {IV }}$ emission profile. The inset is also marked in the spectrum of quasar A with a dotted box. Bottom panel - the thick (red) line is the model of the $\mathrm{Mg}_{\text {II }}+\mathrm{Fe}$ II emission (Vestergaard \& Wilkes 2001) in the wavelength range 4700-5800 $\AA$, supporting the line identification and, hence, our estimate of quasar B redshift. The dotted (green) line shows the underlying continuum.

Table 1. List of the main observed absorption features.

\begin{tabular}{ccccc}
\hline$\lambda$ & $\mathrm{EW}$ & Identification & $\begin{array}{c}\mathrm{EW}_{r} \\
\AA\end{array}$ & Object \\
$\AA$ & $\AA$ & & $\AA$ & \\
$(1)$ & $(2)$ & $(3)$ & $(4)$ & $(5)$ \\
\hline 4547 & $3.8 \pm 1.4$ & $\mathrm{Mg}_{\text {II }} z=0.625 ?$ & $2.3 \pm 0.9$ & $\mathrm{~A}$ \\
5408 & $2.3 \pm 0.7$ & $\mathrm{Mg}_{\text {II }} z=0.932 ?$ & $1.2 \pm 0.4$ & $\mathrm{~A}$ \\
4661 & $2.3 \pm 0.6$ & $\mathrm{Mg}_{\text {II }} z=0.665 ?$ & $1.4 \pm 0.4$ & $\mathrm{~B}$ \\
5833 & $4.4 \pm 1.0$ & $\mathrm{Fe}_{3020} z=0.932 ?$ & $2.3 \pm 0.5$ & $\mathrm{~B}$ \\
7393 & $7.3 \pm 3.3$ & $\mathrm{Fe}_{3} \mathrm{I}_{3830} z=0.932$ & $3.8 \pm 1.7$ & $\mathrm{~B}$ \\
\hline
\end{tabular}

Note. Atmospheric and Galactic lines are dropped. (1) Peak wavelength. (2) Measured EW. (3) Identification. Uncertain classifications are marked with '?'. (4) Rest-frame EW, assuming the redshift in Column (3). (5) Spectrum where the lines are detected.

$\langle\Delta z\rangle \approx 0.5$. Our case represents a record in terms of redshift difference. Other three systems are reported with the nearer quasar at $z<1$, where a detailed study of the host galaxy luminosity and morphology is feasible. The typical projected distances at $z$ (near) are $\sim 60 \mathrm{kpc}$.

A number of apparent quasar pairs have been proposed as anomalous associations (e.g. Burbidge, Hoyle \& Schneider 1997; Galianni et al. 2005) with respect to chance alignments. We estimate that the number of systems reported in Table 2 is consistent with the assumption of chance superposition. In fact, the probability that, given a quasar, a projected companion can be found within a given angular separation $\theta$ follows the Poisson statistics: $P(<\theta) \approx \lambda$. Here, $\lambda$ is the expected number of quasars in the solid angle defined by $\theta, \lambda=\rho(<m) \pi \theta^{2}$, and $\rho(<m)$ is the number density of quasars brighter than a given magnitude $m$. We refer to the $2 \mathrm{dF}$ survey (Croom et al. 2004): $\rho\left(m_{b}<20\right)=13.8$ quasars per square degree, in good agreement with the values from the SDSS (see Yanny et al. 2000). Hence, the probability of finding a quasar with $m_{b} \lesssim 20$ within a 10.5 -arcsec circle is $\sim 4 \times 10^{-4}$.

\section{CONCLUSIONS}

We report the discovery of an apparent quasar pair with angular separation of 10.5 arcsec. Q2225-403A,B is the only apparent pair of quasars for which both the host galaxies have been resolved. Their Ks-band apparent magnitudes $\left[m_{\mathrm{A}}\right.$ (host) $=18.51 ; m_{\mathrm{B}}$ (host) $=$ 17.44] are consistent with those expected for typical quasar host galaxies at the distance indicated by their redshift. The discovery of an intervening absorption system in quasar $\mathrm{A}$ at the same redshift of $\mathrm{B}$ reveals an extended halo around the nearest object. 

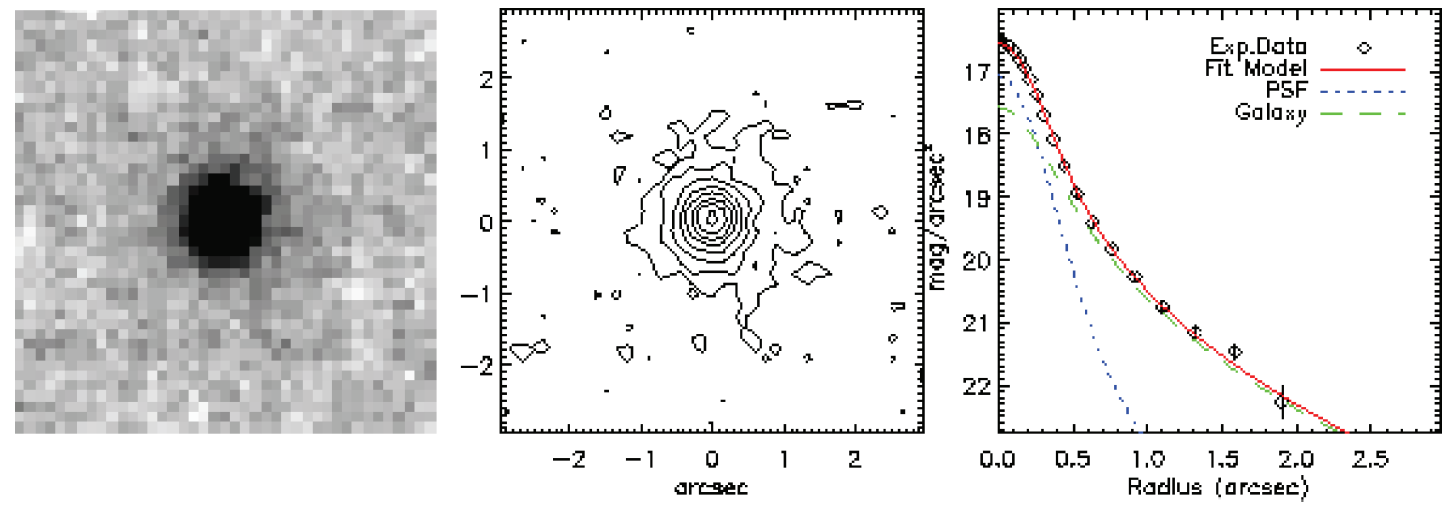

Figure 3. Analysis of the image of Q2225-403B. Left-hand panel - the observed $K_{\mathrm{S}}$-band image of the quasar, centre panel - same image in isophotal contours, right-hand panel - the best-fitting light profile model. See also http://www.dfm.uninsubria.it/astro/qso_host/.

Table 2. Quasar apparent pairs with $\theta<10$ arcsec from the VCV06 catalogue, in a comparison with Q2225-403A,B.

\begin{tabular}{|c|c|c|c|c|c|c|c|c|c|c|c|}
\hline \multicolumn{4}{|c|}{ Nearer quasar } & \multicolumn{4}{|c|}{ Farther quasar } & \multirow{2}{*}{$\begin{array}{c}\theta \\
(\operatorname{arcsec}) \\
(9)\end{array}$} & \multirow{2}{*}{$\begin{array}{l}\text { Proj. sep. } \\
\quad(\mathrm{kpc}) \\
(10)\end{array}$} & \multirow{2}{*}{$\begin{array}{l}\Delta z \\
(11)\end{array}$} & \multirow{2}{*}{$\begin{array}{l}\text { Ref } \\
\text { (12) }\end{array}$} \\
\hline $\begin{array}{c}z \\
(1)\end{array}$ & $\begin{array}{c}\text { RA (J2000) } \\
\text { (2) }\end{array}$ & $\begin{array}{l}\text { Dec. (J2000) } \\
\text { (3) }\end{array}$ & $\begin{array}{l}m_{V} \\
(4)\end{array}$ & $\begin{array}{c}z \\
(5)\end{array}$ & $\begin{array}{c}\text { RA (J2000) } \\
(6)\end{array}$ & $\begin{array}{l}\text { Dec. (J2000) } \\
\text { (7) }\end{array}$ & $\begin{array}{r}m_{V} \\
(8)\end{array}$ & & & & \\
\hline \multicolumn{12}{|c|}{ Known quasar pairs } \\
\hline 1.545 & $00: 02: 12.6$ & $-00: 53: 11$ & 20.46 & 2.206 & 00:02:12.1 & $-00: 53: 09$ & 19.58 & 6.3 & 53 & 0.661 & $c$ \\
\hline 2.030 & 00:40:18.2 & $+00: 55: 31$ & 18.72 & 2.086 & 00:40:18.7 & $+00: 55: 26$ & 19.67 & 7.7 & 64 & 0.056 & $c$ \\
\hline 1.296 & 01:22:12.7 & $+14: 10: 54$ & 19.91 & 1.579 & 01:22:13.1 & $+14: 10: 52$ & 20.03 & 8.1 & 67 & 0.283 & $c$ \\
\hline 1.442 & 02:41:06.9 & $+00: 10: 27$ & 19.89 & 1.673 & 02:41:07.4 & $+00: 10: 28$ & 20.87 & 9.8 & 82 & 0.231 & $c$ \\
\hline 2.180 & 08:14:20.4 & $+32: 50: 16$ & 19.86 & 2.210 & 08:14:19.6 & $+32: 50: 19$ & 20.33 & 9.5 & 78 & 0.030 & $c$ \\
\hline 1.340 & 09:02:35.7 & $+56: 37: 56$ & 20.30 & 1.390 & 09:02:35.4 & $+56: 37: 51$ & 20.63 & 6.6 & 55 & 0.050 & $c, d$ \\
\hline 1.627 & 10:12:15.8 & $-03: 07: 08$ & 18.90 & 2.746 & $10: 12: 15.8$ & $-03: 07: 03$ & 17.60 & 4.5 & 38 & 1.119 & $a, b$ \\
\hline 1.142 & $12: 04: 50.5$ & $+44: 28: 35$ & 19.20 & 1.814 & $12: 04: 50.7$ & $+44: 28: 33$ & 19.42 & 3.8 & 31 & 0.672 & $c, d$ \\
\hline 2.379 & $12: 25: 45.7$ & $+56: 44: 40$ & 19.36 & 2.390 & $12: 25: 45.2$ & $+56: 44: 45$ & 20.51 & 7.4 & 60 & 0.011 & $c$ \\
\hline 2.001 & $12: 49: 48.1$ & $+06: 07: 08$ & 20.42 & 2.376 & $12: 49: 48.2$ & $+06: 07: 13$ & 20.37 & 3.9 & 32 & 0.375 & $c, d$ \\
\hline 0.436 & $15: 50: 43.7$ & $+11: 20: 47$ & 17.23 & 1.901 & $15: 50: 44.0$ & $+11: 20: 47$ & 18.78 & 4.4 & 24 & 1.465 & $a, b$ \\
\hline \multicolumn{12}{|c|}{ New quasar pairs } \\
\hline 1.310 & 00:39:54.3 & $-27: 25: 23$ & 20.61 & 2.100 & 00:39:54.1 & $-27: 25: 14$ & 20.64 & 9.9 & 83 & 0.790 & \\
\hline 1.264 & 00:39:54.8 & $-27: 25: 20$ & 20.26 & 1.310 & $00: 39: 54.3$ & $-27: 25: 23$ & 20.61 & 4.3 & 35 & 0.046 & \\
\hline 1.333 & 01:10:50.8 & $-27: 19: 51$ & 20.10 & 2.261 & 01:10:51.4 & $-27: 19: 57$ & 20.84 & 9.3 & 78 & 0.928 & \\
\hline 1.586 & 03:42:12.4 & $-44: 16: 41$ & 19.20 & 2.077 & $03: 42: 12.6$ & $-44: 16: 36$ & 19.70 & 5.7 & 48 & 0.491 & \\
\hline 1.519 & 10:16:36.3 & $-02: 34: 22$ & 19.55 & 2.617 & 10:16:36.4 & $-02: 34: 12$ & 20.31 & 8.9 & 75 & 1.098 & \\
\hline 0.649 & $10: 51: 26.8$ & $-02: 27: 20$ & 19.43 & 1.160 & $10: 51: 26.3$ & $-02: 27: 17$ & 19.53 & 8.0 & 55 & 0.511 & \\
\hline 0.888 & 11:18:47.9 & $+40: 26: 43$ & 20.70 & 1.129 & 11:18:48.6 & $+40: 26: 47$ & 19.05 & 7.9 & 61 & 0.241 & \\
\hline 1.863 & $23: 33: 05.3$ & $-28: 00: 54$ & 20.33 & 1.970 & $23: 33: 04.7$ & $-28: 00: 55$ & 19.72 & 7.5 & 63 & 0.107 & \\
\hline \multicolumn{12}{|c|}{ This Letter } \\
\hline 0.932 & 22:28:50.4 & $-40: 08: 27$ & 20.20 & 2.410 & 22:28:49.9 & $-40: 08: 34$ & 20.10 & 10.5 & 83 & 1.478 & \\
\hline
\end{tabular}

Note. (1-4) $-z$, RA, Dec. and $m_{V}$ of the quasar with the lower $z$. (5-8)- the same for the higher $z$ quasar. (9) - angular separation. (10) projected separation assuming the redshift of the lower $z$ quasar. (11) - redshift difference. (12) - references (only those references concerning quasar pairs are included): $a$ - Burbidge et al. (1997); $b$ - Sluse et al. (2003); $c$ - Hennawi et al. (2006) and $d$ - Myers et al. (2007).

Based on the known surface density distribution of quasars, we find that the a priori probability of finding such a pair in our survey is of the order of 0.6 per cent and the discovery of the absorption system on the spectrum of quasar A at the same $z$ of quasar B is a clear evidence that these two objects are an apparent pair.

We propose a list of apparent quasar pairs which deserve a specific study to investigate the properties of the extended halo around quasar host galaxies.

\section{ACKNOWLEDGMENTS}

We thank Marzia Labita for useful discussions. This work was based on observations made with the ESO/3.6-m telescope in La Silla and with the ESO/VLT in Paranal. This research has made use of the
Vizier Service, available at http://vizier.u-strasbg.fr/viz-bin/VizieR and of the NED which is operated by the Jet Propulsion Laboratory, California Institute of Technology, under contract with the National Aeronautics and Space Administration.

\section{REFERENCES}

Adelman-McCarthy J. K. et al., 2008, ApJS, 175, 297

Bowen D. V. et al., 2006, ApJ, 645, L105

Burbidge G., Hoyle F., Schneider P., 1997, A\&A, 320, 8

Chieregato M., Miranda M., Jetzer P., 2007, A\&A, 474, 777

Croom S. M., Schade D., Boyle B. J., Shanks T., Miller L., Smith R. J., 2004, ApJ, 606, 126

D’Odorico V., Bruscoli M., Saitta F., Fontanot F., Viel M., Christiani S., Monaco P., 2008, MNRAS, 389, 1727 
Foreman G., Volonteri M., Dotti M., 2009, ApJ, 693, 1554

Francis P. J., Hewett P. C., Foltz C. B., Chaffee F. H., Weymann R. J., Morris S. L., 1991, ApJ, 373, 465

Galianni P., Burbidge E. M., Arp H., Junkkarinen V., Burbidge G., Zibetti S., 2005, ApJ, 620, 88

Gallerani S., Ferrara A., Fan X., Choudhury T. R., 2008, MNRAS, 386, 359

Hennawi J. F., Prochaska J. X., 2007, ApJ, 655, 735

Hennawi J. F. et al., 2006, AJ, 131, 1

Hewitt A., Burbidge G., 1993, ApJS, 87, 451

Jakobsen P., Perryman M. A. C., di Serego Alighieri S., Ulrich M. H., Macchetto F., 1986, ApJ, 303, L27

Kacprzak G. G., Churchill C. W., Steidel C. C., Murphy M. T., Evans J. L., 2007, ApJ, 662, 909

Kang E., Im M., 2009, ApJ, 691, L33

Komberg B. V., Kravtsov A. V., Lukash V. N., 1996, MNRAS, 282, 713

Kotilainen J. K., Falomo R., Decarli R., Treves A., Uslenghi M., Scarpa R. 2009, ApJ, submitted (K09)

Mannucci F., Basile F., Poggianti B. M., Cimatti A., Daddi E., Pozzetti L., Vanzi L., 2001, MNRAS, 326, 745
Moorwood A. et al., 1998, ESO Messenger, 94, 7

Myers A. D., Brunner R. J., Richards G. T., Nichol R. C., Schneider D. P., Bahcall N. A., 2007, ApJ, 658, 99

Prochaska J. X., Hennawi J. F., 2009, ApJ, 690, 1558

Richards G. T. et al., 2006, AJ, 131, 2766

Schlegel D. J., Finkbeiner D. P., Davis M., 1998, ApJ, 500, 525

Schirber M., Miralda-Escudé J., McDonald P., 2004, ApJ, 610, 105

Shen Y. et al., 2008, preprint (arXiv:0810.4144)

Sluse D., Surdej J., Claeskens J.-F., De Rop Y., Lee D. W., Iovino A., Hawkins M. R. S., 2003, A\&A, 397, 539

Veron-Cetty M. P., Veron P., 2006, A\&A, 455, 773 (VCV06)

Vestergaard M., Wilkes B. J., 2001, ApJS, 134, 1

Wittman D. M., Tyson J., Kirkman D., Dell'Antonio I., Bernstein G., 2000, Nat, 405, 143

Yanny B. et al., 2000, ApJ, 540, 825

This paper has been typeset from a $\mathrm{T}_{\mathrm{E}} \mathrm{X} / \mathrm{LT}_{\mathrm{E}} \mathrm{X}$ file prepared by the author. 